\title{
Evidence of effectiveness of clinical audit in improving histopathology reporting standards of mastectomy specimens
}

\author{
M A C Appleton, A G Douglas-Jones, J M Morgan
}

\begin{abstract}
Aim-To assess the effectiveness of clinical audit in improving standards in histopathological reporting of mastectomy specimens.

Methods-Reports on mastectomy specimens containing tumour issued by nonspecialist histopathologists in 1990, 1992, 1994, and 1996 were scored for their information content. There were 10 reports evaluated from each year. Before 1990 no reporting guidelines had been formulated within the department. The audits in 1992 and 1994 were performed after agreed written guidelines (including the establishment of six essential pieces of information), and in 1996 the specimens were reported using a proforma.

Results-There was a significant increase in information after the introduction of written guidelines but there was a reduction in information over time. In 1990 none of the 10 reports included all six pieces of mandatory information; in 1992 four of the reports contained all mandatory information; in 1994 only one report contained all mandatory information. The introduction of a proforma for reporting resulted in further significant improvement with all 10 reports in 1996 containing all mandatory information.

Conclusions-Successive rounds of audit increases the standard of reporting in histopathology. There is a need for continuing monitoring of standards as these may deteriorate over time. Reporting complex specimens on a proforma has a significant beneficial effect on information content. (f Clin Pathol 1998;51:30-33)
\end{abstract}

Keywords: audit; breast cancer; reporting

Clinical audit has been introduced into the routine practice of medicine over the past five years. This has meant extra work for many clinicians who often regard the process with a degree of scepticism. The principle of auditing clinical practice is sound. Medical practitioners assess aspects of their current clinical practice and set standards that should be achieved in every case. These standards are documented for reference during ongoing clinical practice. Practice is subsequently reviewed and the performance of the clinical team is assessed in relation to the documented standards. If practice falls short of the agreed standards, changes are implemented to achieve compliance. This process is cyclical and iterative and designed to improve clinical practice and maintain agreed standards over time. The prime objective of this activity is to monitor patient management and to ensure that each patient receives the quality of clinical care that is laid out in documented guidelines. ${ }^{1}$

Audit in our department has taken the form of examination of groups of histological reports of a particular specimen type for completeness of information content. After the initial audit session guidelines are produced for the reporting of these specimens (setting the standard) and these guidelines or standards are circulated to all members of the department. Subsequently a similar group of specimens that have been handled by the department since the production of guidelines are reviewed and the content is compared with the set standards and the previous audit results. ${ }^{2}$

In the past decade there has been a rapid increase in the amount of prognostically and clinically important information that is demanded by clinical colleagues for patient management. This increase in workload is reflected in the number pieces of information and number of words in histological reports. ${ }^{3}$ Histopathologists have to remember to include an increasing array of important diagnostic and prognostic observations. Recent regionally based studies have indicated that not all reports issued by non-specialist histopathologists contain information essential for clinical management. ${ }^{56}$

We report on the results of clinical audit over a six year period related to mastectomies containing tumour for which a large amount of important prognostic information is routinely required. We examined the information content of reports on specimens handled routinely and not from patients within the National Health Service Breast Screening Programme (NHSBSP), and therefore not reported by a pathologist with a special interest in breast pathology. The NHSBSP was set up in 1987 and at that time a national coordinating group for breast screening pathology issued formal guidelines for the reporting of screen detected breast lesions. ${ }^{7}$ Hence formal guidelines were available for the reporting of mastectomy specimens with tumour at the time of the first audit reported here.

Methods

SAMPLING

Reports on 40 mastectomy specimens containing invasive tumour with axillary node dissections were identified-10 cases each from 
Table 1 Percentage of reports containing specified information from four audit cycles

\begin{tabular}{|c|c|c|c|c|c|}
\hline Information & Score & 1990 & 1992 & 1994 & 1996 \\
\hline \multicolumn{6}{|l|}{ Macroscopic description } \\
\hline Nature of specimen & 3 & 40 & 60 & 40 & 100 \\
\hline Side of mastectomy & 2 & 20 & 60 & 50 & 100 \\
\hline Size of specimen & 2 & 100 & 100 & 90 & 100 \\
\hline \multicolumn{6}{|l|}{ Quadrant of breast in which tumour was } \\
\hline found & 2 & 90 & 80 & 60 & 100 \\
\hline Size of overlying skin & 1 & 90 & 100 & 100 & 100 \\
\hline Distance of tumour from nipple & 1 & 10 & 50 & 30 & 100 \\
\hline Description of cut surface of the tumour & 1 & 80 & 80 & 80 & 10 \\
\hline Description of skin & 1 & 50 & 0 & 20 & 0 \\
\hline Description of nipple & 1 & 20 & 50 & 50 & 100 \\
\hline $\begin{array}{l}\text { Presence or absence of fascia or skeletal } \\
\text { muscle }\end{array}$ & 1 & 30 & 0 & 0 & 10 \\
\hline \multicolumn{6}{|l|}{ Microscopic description } \\
\hline Type of tumour & 3 & 80 & 100 & 100 & 100 \\
\hline Grade of tumour (Bloom and Richardson) & 3 & 40 & 90 & 80 & 100 \\
\hline Size of tumour & 3 & 40 & 80 & 70 & 100 \\
\hline \multicolumn{6}{|l|}{ Lymph nodes (number involved and number } \\
\hline sampled) & 3 & 100 & 100 & 100 & 100 \\
\hline Involvement of the resection margin & 3 & 60 & 90 & 90 & 100 \\
\hline Presence or absence of vascular invasion & 2 & 60 & 90 & 60 & 100 \\
\hline Involvement of apical lymph node & 2 & $50(3 / 6)$ & $40(2 / 5)$ & 80 & 80 \\
\hline Distance of tumour to resection margins & 2 & 30 & 100 & 80 & 100 \\
\hline Presence or absence of concurrent DCIS & 1 & 70 & 100 & 70 & 100 \\
\hline Size of concurrent DCIS & 1 & 0 & $38(3 / 8)$ & $33(3 / 9)$ & 100 \\
\hline Description of nipple & 1 & 50 & 70 & 50 & 60 \\
\hline Description of background breast & 1 & 70 & 90 & 90 & 80 \\
\hline Summary provided & 1 & 90 & 100 & 80 & 100 \\
\hline
\end{tabular}

DCIS, ductal carcinoma in situ.
Table 2 Scores for individual report information content related to guideline score

\begin{tabular}{lllll}
\hline Case & 1990 & 1992 & 1994 & 1996 \\
\hline 1 & $10 / 41$ & $33 / 38$ & $27 / 40$ & $36 / 41$ \\
2 & $30 / 41$ & $25 / 41$ & $28 / 41$ & $36 / 41$ \\
3 & $27 / 41$ & $36 / 39$ & $34 / 41$ & $37 / 40$ \\
4 & $22 / 40$ & $32 / 39$ & $30 / 41$ & $36 / 41$ \\
5 & $29 / 39$ & $32 / 41$ & $27 / 41$ & $37 / 40$ \\
6 & $19 / 38$ & $28 / 41$ & $33 / 41$ & $38 / 41$ \\
7 & $18 / 39$ & $29 / 41$ & $27 / 41$ & $37 / 41$ \\
8 & $25 / 41$ & $33 / 38$ & $26 / 41$ & $37 / 40$ \\
9 & $22 / 39$ & $32 / 39$ & $28 / 41$ & $39 / 41$ \\
10 & $28 / 40$ & $32 / 41$ & $28 / 41$ & $35 / 40$ \\
Mean total score & 23.0 & 31.3 & 28.8 & 36.8 \\
\hline
\end{tabular}

$1990 v 1992, \mathrm{~S}$ value $5.92 ; \mathrm{p}=0.001$.

$1992 v 1994, \mathrm{~S}$ value $2.53 ; \mathrm{p}=0.25$.

$1994 v 1996, \mathrm{~S}$ value $6.83 ; \mathrm{p}=0.001$

$1990 v 1994, S$ value $3.40 ; p=0.05$

$1990 v 1996, \mathrm{~S}$ value $10.17 ; \mathrm{p}=0.001$.

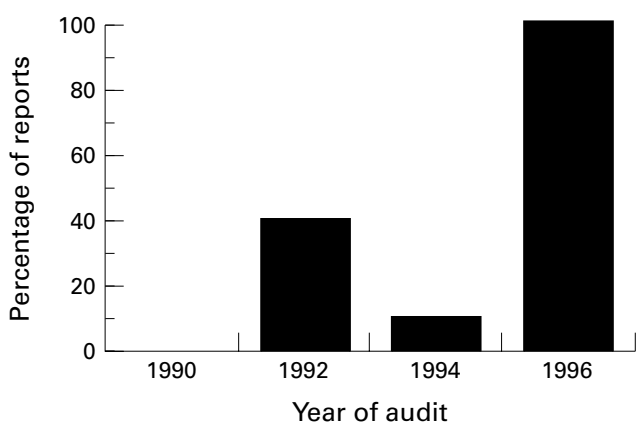

from 1990 was reported before the production of departmental guidelines. The second group, from 1992, was audited soon after the dissemination of the guidelines. The third group, from 1994, formed a continuing audit to ensure maintenance of standards. The fourth group, from 1996, was after the introduction of a departmental proforma for the reporting of breast specimens. The proforma was based on the NHSBSP guidelines and was used in reporting the 10 cases from 1996.

AUDIT FOR INFORMATION CONTENT

Reports were scrutinised for information under three headings: macroscopic description, microscopic description, and summary (table 1 ).

SETTING OF STANDARDS

Standards were set at the first audit meeting at which a consultant surgeon with a special interest in breast surgery was present who identified six pieces of information that he felt were essential in the report for clinical management: nature of specimen, type of tumour, grade of tumour, size of tumour, lymph node involvement, resection margin involvement.

SCORING OF INFORMATION IN REPORTS

For the purpose of this study we devised a weighted points system for each of the items of information that should be included in the report allowing a total score to be attributed to each report (table 1). Mandatory information items score three points, important information items score two points, and lesser items score one point. The total possible score varied between cases as some of the information was not relevant in each case-for example, if there was no ductal carcinoma in situ no size could be recorded.
Figure 1 Percentage of reports containing all mandatory information.

\section{STATISTICAL ANALYSIS}

Differences between the 10 proportions in each of the four audit groups were compared using Scheffe's parametric multiple contrast procedure ( $\mathrm{S}$ test). The null hypothesis is that there is no significant contrast between one group of proportions and another. The application of Scheffe's $S$ test to the data presented used Freeman-Tukey angular transformations for the individual proportions and a contrast coefficient of 0.1 for four groups of 10 proportions per group.

\section{Results}

Each report was scored out of a maximum of 38-41 points and the individual and mean scores are shown in (table 2). The Scheffe's S values are a direct measure of the relative improvement, or lack of it, between the years studied. This improvement is reflected in the numerical difference between the experimentally determined $S$ value and the calculated critical value $S$. There was a significant improvement $(S=5.92 ; p=0.001)$ in score between 1990 and 1992 after the introduction of guidelines and a reduction in score between 1992 and 1994 that was not significant $(\mathrm{S}=2.53 ; \mathrm{p}=0.25)$. Despite this deterioration in performance, the scores in 1994 were significantly better than before departmental guidelines (1990 $v 1994 ; \mathrm{S}=3.40 ; \mathrm{p}=0.05)$. There was a further significant improvement in scores in 1996 after the introduction of a proforma (1994 v 1996; $S=6.83 ; p=0.001)$ (table 2).

Figure 1 shows the number of reports that contained all six of the mandatory pieces of information defined at the first audit. In 1990 
none of 10 reports contained all the information required. Four of 10 reports contained all six pieces of information after the issuing of reporting guidelines. All 10 cases reported by proforma in 1996 contained all mandatory information.

\section{Discussion}

The purpose of this study was to assess the effectiveness of clinical audit in a histopathology department. Mastectomies containing tumour were chosen as a well defined group of cases requiring prognostically important information and where reasonable numbers of cases were available. All specimens were reported outside the NHSBSP by non-specialist histopathologists. The 10 cases audited in 1990 were obtained over four months, those from 1996 took over 10 months to accrue. Despite the perception that these cases would be quite common in a large centre handling some 600 breast specimens each year, the numbers identified were quite small. Reasons for this decreasing frequency include the trend towards conservative management of breast cancer resulting in wide local excision specimens unsuitable for inclusion in this study. Many carcinomas are now diagnosed and treated within the NHSBSP, and these were excluded from this study. The NHSBSP has had quality assessment of reporting standards from its inception and represents a separate group of cases to those reported by non-specialist histopathologists. The concentration on nonscreened cases reflects more accurately general histopathology practice. A further advantage of selecting this specimen type is that, at the beginning of clinical audit, guidelines for the reporting of screen detected breast lesions had been drawn up by a national coordinating group for breast screening pathology. The results of the first audit indicated that these guidelines were not being used by general pathologists in routine practice. Early audit sessions in our department revealed a large number of instances where comments on particular features were omitted. Commonly this was because the feature was not present and therefore not considered worthy of comment, although it may have been looked for. Features such as the presence or absence of vascular invasion and involvement or not of resection margins by tumour were examples of these sins of omission. In formulating the original guidelines it was decided that statements, either positive or negative, should be made in each report to indicate that the feature had been looked for.

There was considerable discussion at early audit meetings as to what information should go into the report and what information was clinically essential. A balance needed to be achieved between an all embracing council of perfection and a reductionist approach where only those items essential for clinical management were included in the report. The guidelines issued after the 1990 audit broadly followed the NHSBSP standard. The local guidelines were circulated to all members of the department and resulted in a significant improvement $(p=0.001)$ in the information content of reports in 1992.

Two years later, in 1994, the performance of the department had deteriorated despite the continuing presence of guidelines; however, the decline was not significant. There are a number of possible reasons for this deterioration. Guidelines tend to get filed away and over a period of time pathologists lose them or cease to refer to them. In a large teaching hospital department there is a constant turnover of junior staff and it is possible that new appointees are not made aware of the existence of reporting guidelines. This reduction in the level of reporting over a two year period illustrates the importance of continuing rounds of audit to check that standards are maintained, that guidelines are reviewed and modified, and that pathologists are reminded of the standards that have been set. Not infrequently, surgeons write to reporting pathologists requesting a specific piece of information if it is omitted from the report underscoring their clinical importance. The NHSBSP has used a reporting proforma from its inception and Leslie and Rosai have proposed the use of templates or checklists as a means of achieving standard information content. ${ }^{8}$

As can be seen from fig 1, the effect of introducing a written proforma has been dramatic. A preprinted proforma is attached to all request forms for pathological examination of breast specimens as they arrive in the department. This proforma includes formal prompts for macroscopic and microscopic features with free text areas for additional descriptions. All the mandatory information is included in the formal part of the proforma. Interestingly a description of the cut surface of the tumour is not specifically requested but is left to the discretion of the pathologist to fill in the free text area. The results from the 1996 audit show that this option is rarely used. In the microscopic description a free text comment on background breast and nipple histology is prompted, but there are no particular boxes identified for these items. The 1996 audit shows that these microscopic descriptions, although prompted, are not universally supplied. These features illustrate the strengths and weaknesses of the proforma format. Clearly, the provision of prompt boxes for items such as tumour type, size, grade and so on have resulted in all reports containing the six mandatory pieces of clinical information. However, if the proforma does not specifically provide a prompt or response box that feature is likely to be ignored or poorly reported.

The introduction of proforma reporting has highlighted the council of perfection nature of many of these documents. When groups of experts with special interest come together there is a tendency to demand information that, although of pathological and academic interest, may not be of practical value in the routine clinical situation. This point has been repeatedly raised at audit meetings. In the current climate of continuing reduction in resources (cost improvement programmes), 
increasing workload, and information content required, pathologists are legitimately asking "why are we reporting this particular feature and what is the evidence that observation of this feature has prognostic or management implications?". This could lead to a reductionist pressure in which reports will simply consist of a list of the pathological features of proven prognostic importance. Resolution of this may lie in the more rigorous application of evidence-based medicine.

In this study we have documented the effect of clinical audit on the routine reporting practice for a particular specimen type with a high information content. We have provided evidence that successive rounds of iterative audit with setting of standards and comparison of reports with those standards has a significant effect on the reporting of important pathological features in histopathology practice. There is evidence that it is important to repeat the audit process to maintain reporting standards. We have also shown that the introduction of a proforma style of reporting can produce a dramatic result with $100 \%$ of reports containing all mandatory clinical information. The experience with proforma reporting suggests that there may be too much detailed information being requested that may be unnecessary for clinical management, and that there may be a reduction in the use of free text to describe cut surface and other histopathological features even though a prompt may be present.

1 Crombie IK, Davies HTO, Abraham SCS, et al. The audit handbook: improving health care through clinical audit. Chichester: John Wiley and Sons, 1993.

2 Campbell F, Griffiths DFR. Quantitative audit of the content of histopathological reports. F Clin Pathol 1994;47: content $360-1$.

3 Cross SS, Bull AD. Is the informational content of histopathological reports increasing? F Clin Pathol 1992;45: 179-80.

4 Parham DM. The hidden increase in histopathologists' workload. F Clin Pathol 1996;49:689-90.

5 Bull AD, Biffin AHB, Mella J, et al. Colorectal cancer pathology reporting: a regional audit. $\mathcal{F}$ Clin Pathol 1997;50:138-42.

6 Miller JM, Slader DN. Do histopathology reports of primary cutaneous melanoma contain enough essential primary cutaneous melanoma contain en

7 Royal College of Pathologists Working Group. Pathology reporting in breast cancer screening. Sheffield: NHSBSP Publications, 1989.

8 Leslie KO, Rosai J. Standardization of the surgical pathology report: formats, templates and synoptic reports. Semin Diagn Pathol 1994;11:253-7. 